\title{
Pacific
}

Journal of

Mathematics

\section{SINGULARITIES OF THE PROJECTIVE DUAL VARIETY}

\author{
ROLAND ABUAF
}

Volume $253 \quad$ No. 1 


\title{
SINGULARITIES OF THE PROJECTIVE DUAL VARIETY
}

\author{
ROLAND ABUAF
}

\begin{abstract}
Let $X \subset \mathbb{P}^{N}$ be an irreducible, nondegenerate projective variety and let $X^{*} \subset \mathbb{P}^{N^{*}}$ be its projective dual. Let $L \subset \mathbb{P}^{N}$ be a linear space such that $\left\langle L, T_{X, x}\right\rangle \neq \mathbb{P}^{N}$ for all $x \in X_{\text {smooth }}$ and such that the lines in $X$ meeting $L$ do not cover $X$. If $x \in X$ is general, we prove that the multiplicity of $X^{*}$ at a general point of $\left\langle L, T_{X, x}\right\rangle^{\perp}$ is strictly greater than the multiplicity of $X^{*}$ at a general point of $L^{\perp}$. This is a strong refinement of Bertini's theorem.
\end{abstract}

\section{Introduction}

1.1. Multiplicities of the projective dual. Let $X \subset \mathbb{P}^{N}$ be an irreducible projective variety over the field of complex numbers. Let $X^{*} \subset \mathbb{P}^{N^{*}}$ be its projective dual, let $L \subset \mathbb{P}^{N}$ be a linear space and $H$ be a general hyperplane containing $L$. Bertini's classical theorem asserts that the tangency locus of $H$ with $X$ is included in $X \cap L$. Very little is known about the hyperplanes whose tangency locus with $X$ lies outside $L \cap X$. It is tempting to think that the multiplicity in $X^{*}$ of such a hyperplane is strictly larger than the multiplicity of a general hyperplane containing $L$. The following example shows that this is not true for every $L$.

Example 1.1.1. Let $X \subset \mathbb{P}^{4}$ be a smooth hyperplane section of $\mathbb{P}^{1} \times \mathbb{P}^{2} \subset \mathbb{P}^{5}$. The variety $X$ is a ruled surface of degree 3 . Its dual is a hypersurface of degree 3 in $\mathbb{P}^{4 *}$ which does not contain any points of multiplicity higher than 2 . Let $L$ be the exceptional section of $X$. If $H \subset \mathbb{P}^{4}$ is a general hyperplane which contains $L$, then $H \cap X=L \cup D_{1} \cup D_{2}$, where $D_{1}$ and $D_{2}$ are two distinct lines on $X$ such that $D_{1} . D_{2}=0$ and $L . D_{i}=1$ for $i=1,2$. As a consequence, a general point of $L^{\perp}$ is of multiplicity 2 in $X^{*}$. Now, let $D \subset X$ be a line such that $D . L=1$ and let $x \in D$ such that $x \notin L$. The hyperplane containing $L$ and $T_{X, x}$ is a point of multiplicity exactly 2 in $X^{*}$, that is, the multiplicity of a general point of $L^{\perp}$.

This example shows that, even for general $x \in X$, the multiplicity in $X^{*}$ of a hyperplane containing $L$ and tangent to $X$ at $x$ may well be equal to the multiplicity of a general hyperplane containing $L$. Thus, without extra hypotheses on $L$, it

MSC2000: 14B05, 14N15.

Keywords: projective geometry, singularities, dual variety. 
seems hopeless to say something about the multiplicity in $X^{*}$ of special points of $L^{\perp}$. For this purpose, we introduce a definition:

Definition 1.1.2. Let $X \subset \mathbb{P}^{N}$ be an irreducible projective variety and let $L \subset \mathbb{P}^{N}$ be a linear space. Consider the conormal diagram

$$
\begin{array}{ccc}
\swarrow^{q} & I\left(X / \mathbb{P}^{N}\right):=\overline{\left\{(H, x) \in \mathbb{P}^{*} \times X_{\text {smooth }}: T_{X, x} \subset H\right\}} & \\
X^{*} \subset \mathbb{P}^{N^{*}} & \searrow \\
& X \subset \mathbb{P}^{N}
\end{array}
$$

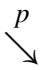

Let $F_{1}, \ldots, F_{m}$ be all the irreducible components of $q^{-1}\left(L^{\perp}\right)$ such that the restrictions

$$
\left.q\right|_{F_{i}}: F_{i} \rightarrow L^{\perp}
$$

are surjective. The contact locus of $L$ with $X$, which we denote by $\operatorname{Tan}(L, X)$, is the union of the $p\left(F_{i}\right)$, for $1 \leq i \leq m$.

In the case where $L$ is a hyperplane, the contact locus $\operatorname{Tan}(L, X)$ is called the tangency locus of $L$ with $X$. A tangent hyperplane to $X$ is a hyperplane $H \subset \mathbb{P}^{N}$ such that $\operatorname{Tan}(H, X) \neq \varnothing$.

The contact locus $\operatorname{Tan}(L, X)$ can be thought as the variety covered by the tangency loci of general hyperplanes containing $L$. In case $L^{\perp} \not \subset X^{*}$, this locus is empty. We always have the inclusion

$$
\overline{\left\{x \in X_{\text {smooth }}: T_{X, x} \subset L\right\}} \subset \operatorname{Tan}(L, X),
$$

but if $\operatorname{dim}(L)<N-1$ or if $X$ is not smooth, the former locus can be strictly smaller than the latter. Note also that Bertini's theorem says that $\operatorname{Tan}(L, X) \subset L \cap X$. Finally, the contact locus is well behaved. If for a general hyperplane $H^{\prime}$ containing $L$, we have $\operatorname{dim} \operatorname{Tan}\left(H^{\prime}, X\right)>0$, then

$$
\operatorname{Tan}(H \cap L, H \cap X)=H \cap \operatorname{Tan}(L, X),
$$

for any general hyperplane $H \subset \mathbb{P}^{N}$.

Example 1.1.3. If $X \subset \mathbb{P}^{N}$ is such that $X^{*}$ is a hypersurface and $L=T_{X, x}$, where $x \in X$ is a general point, then $\operatorname{Tan}(L, X)=x$.

- If $X=G(1,7) \subset \mathbb{P}^{27}$ and $L=\left\langle T_{X, y_{1}}, T_{X, y_{2}}\right\rangle$, where $y_{1}, y_{2} \in \mathbb{G}(1,7)$ are two general points, then $\operatorname{Tan}(L, X)=\left\{x \in X: T_{X, x} \subset L\right\}$ is a 4-dimensional quadric, the entry locus of a general point $z \in\left\langle y_{1}, y_{2}\right\rangle$.

- If $X=G(1,4) \subset \mathbb{P}^{9}$ and $L=T_{X, y}$, for any $y \in X$, then $\operatorname{dim} \operatorname{Tan}(L, X)>0$, whereas $\left\{x \in X: T_{X, x} \subset L\right\}=\{y\}$. 
Definition 1.1.4. Let $X \subset \mathbb{P}^{N}$ be an irreducible projective variety, and let $L \subset \mathbb{P}^{N}$ be a linear subspace. The shadow of $L$ on $X$, which we denote by $\operatorname{Sh}_{X}(L)$, is the closed variety covered by the linear spaces $M \subset X$ such that $\operatorname{dim}(M)=\operatorname{def}(X)+1$ and $\operatorname{dim}(M \cap \operatorname{Tan}(L, X))=\operatorname{def}(X)$.

Here $\operatorname{def}(X)=\operatorname{codim}\left(X^{*}\right)-1$. The shadow is also well behaved. Namely, assume that $\operatorname{def}(X)>0$; then

$$
\operatorname{Sh}_{L}(X)=X \Longleftrightarrow \operatorname{Sh}_{H \cap L}(H \cap X)=H \cap X,
$$

for any general hyperplane $H \subset \mathbb{P}^{N}$. Note also that if $x \in X$ is a general point and $L=T_{X, x}$, then $\operatorname{Sh}_{L}(X) \neq X$, unless $X$ is a linear space. Indeed, if $X^{*}$ is a hypersurface, this is obvious since $\operatorname{Tan}\left(T_{X, x}, X\right)=x$ for general $x \in X$. If $X^{*}$ is not a hypersurface, take enough general hyperplane sections of $X$ passing through $x$, so that the corresponding dual is a hypersurface.

Main Theorem 1.1.5. Let $X \subset \mathbb{P}^{N}$ be an irreducible, nondegenerate projective variety. Let $L \subset \mathbb{P}^{N}$ be a linear space such that $\operatorname{Sh}_{X}(L) \neq X$. Then, for all $x \in$ $X_{\text {smooth }}$ such that $x \notin \operatorname{Sh}_{X}(L)$ and such that $\left\langle L, T_{X, x}\right\rangle \neq \mathbb{P}^{N}$, the multiplicity in $X^{*}$ of a general hyperplane containing $\left\langle L, T_{X, x}\right\rangle$ is strictly larger than the multiplicity in $X^{*}$ of a general hyperplane containing $L$.

If $X$ is the ruled cubic surface considered in Example 1.1.1 and $L$ is the directrix of $X$, one notices easily that $\operatorname{Sh}_{X}(L)=X$. This shows that the hypothesis $\operatorname{Sh}_{X}(L) \neq$ $X$ can not be withdrawn. Here is an obvious consequence of Main Theorem 1.1.5:

Corollary 1.1.6. Let $X \subset \mathbb{P}^{N}$ be an irreducible, nondegenerate projective variety. Let $L \subset \mathbb{P}^{N}$ be a linear space such that $\left\langle L, T_{X, x}\right\rangle \neq \mathbb{P}^{N}$ for general $x \in X$, and such that the lines in $X$ meeting $L$ do not cover $X$. Then, for general $x \in X$, the multiplicity in $X^{*}$ of a general hyperplane containing $\left\langle L, T_{X, x}\right\rangle$ is strictly larger than the multiplicity in $X^{*}$ of a general hyperplane containing $L$.

\subsection{Variety of multisecant spaces and duals.}

Definition 1.2.1. Let $X \subset \mathbb{P}^{N}$ be an irreducible projective variety. Let $S_{X}^{k}=\left\{\left(x_{0}, \ldots, x_{k}, u\right) \in X \times \cdots \times X \times \mathbb{P}^{N}: \operatorname{dim}\left\langle x_{0}, \ldots, x_{k}\right\rangle=k, u \in\left\langle x_{0}, \ldots x_{k}\right\rangle\right\}$, and let $S_{X}^{k}$ be its Zariski closure in $X \times \cdots \times X \times \mathbb{P}^{N}$. Denote by $\phi$ the projection onto $\mathbb{P}^{N}$. The variety $S^{k}(X)=\phi\left(S_{X}^{k}\right)$ is the $k$-th secant variety to $X$.

Theorem 1.2.2 (Terracini's lemma [Zak 1993]). Let $X \subset \mathbb{P}^{N}$ be an irreducible projective variety, and let $\left(x_{0}, \ldots, x_{k}\right) \in X \times \cdots \times X$, be general points. If $u$ is general in $\left\langle x_{0}, \ldots, x_{k}\right\rangle$, we have the equality

$$
\left\langle T_{X, x_{0}}, \ldots, T_{X, x_{k}}\right\rangle=T_{S^{k}(X), u} .
$$


Definition 1.2.3. Let $X \subset \mathbb{P}^{N}$ be an irreducible, nondegenerate projective variety, and let $k$ be an integer such that $S^{k}(X) \neq \mathbb{P}^{N}$. We say that $X$ is dual $k$-defective if $\operatorname{def}\left(S^{k}(X)\right)>t\left(S^{k}(X)\right)$, where $t\left(S^{k}(X)\right)$ is the dimension of the general fiber of the Gauss map of $S^{k}(X)$.

Note that when $X$ is smooth, then dual 0-defectiveness is the classical dual defectiveness. I don't know if there exist smooth varieties which are dual $k$-defective for some $k \geq 1$, but which are not dual 0-defective. I believe it would be interesting to find some examples of such varieties.

Note also that the notion of dual $k$-defectiveness seems to be related to that of $R_{k}$ regularity explored in [Chiantini and Ciliberto 2010].

Here is a consequence of the Main Theorem 1.1.5 and Terracini's lemma:

Proposition 1.2.4. Let $X \subset \mathbb{P}^{N}$ be an irreducible, nondegenerate, smooth, projective variety. Assume moreover that for all $k$ such that $S^{k}(X) \neq \mathbb{P}^{N}$ the variety $X$ is not dual $k-1$-defective. Then, for any such $k$, we have

$$
S^{k}(X)^{*} \subset X_{k+1}^{*}
$$

where $X_{k+1}^{*}$ is the set of points which have multiplicity at least $k+1$ in $X^{*}$.

Proof. The case $k=0$ is the definition of $S^{0}(X)^{*}=X^{*}$. Let $k \geq 1$ be an integer such that $S^{k}(X) \neq \mathbb{P}^{N}$, let $z \in S^{k-1}(X)$ be a general point and $H$ be a general hyperplane containing $T_{S^{k-1}(X), z}$. Let's prove that

$$
\operatorname{Tan}(H, X)=\left\{x \in X: T_{X, x} \subset T_{S^{k-1}(X), z}\right\} .
$$

Let $x_{0}, \ldots, x_{k-1}$ be $k$ general points in $\operatorname{Tan}(H, X)$. Let $z^{\prime}$ be a general point in $\left\langle x_{0}, \ldots, x_{k-1}\right\rangle$, by Terracini's lemma we have

$$
T_{S^{k-1}(X), z^{\prime}}=\left\langle T_{X, x_{0}}, \ldots, T_{X, x_{k-1}}\right\rangle .
$$

So $z^{\prime} \in \operatorname{Tan}\left(H, S^{k-1}(X)\right)$. But $\operatorname{def}\left(S^{k-1}(X)\right)=t\left(S^{k-1}(X)\right)$ by hypothesis, and this implies

$$
z^{\prime} \in \overline{\left\{y \in S^{k-1}(X)_{\text {smooth }}: T_{S^{k-1}(X), y}=T_{S^{k-1}(X), z}\right\}},
$$

so that $x_{0}, \ldots, x_{k-1} \in\left\{x \in X: T_{X, x} \subset T_{S^{k-1}(X), z}\right\}$.

We now prove that $\operatorname{Sh}_{X}\left(T_{S^{k-1}(X), z}\right) \neq X$. The argument above shows that

$$
\operatorname{Tan}\left(T_{S^{k-1}(X), z}, X\right)=\left\{x \in X: T_{X, x} \subset T_{S^{k-1}(X), z}\right\} .
$$

Assume $\operatorname{Sh}_{X}\left(T_{S^{k-1}(X), z}\right)=X$. For all $x^{\prime \prime} \in X$, there is $x^{\prime} \in\left\{x \in X: T_{X, x} \subset T_{S^{k}(X), z}\right\}$ such that the line $\left\langle x^{\prime \prime}, x^{\prime}\right\rangle$ lies in $X$. But since $X$ is smooth, this line $\left\langle x^{\prime \prime}, x^{\prime}\right\rangle$ lies in $T_{X, x^{\prime}}$. So we have $X \subset T_{S^{k-1}(X), z}$, which contradicts the nondegeneracy. 
As a consequence of Main Theorem 1.1.5, we get that for a general $x \in X$, the multiplicity in $X^{*}$ of a general hyperplane containing $\left\langle T_{S^{k-1}(X), z}, T_{X, x}\right\rangle$ is strictly larger than the multiplicity in $X^{*}$ of a general hyperplane containing $T_{S^{k-1}(X), z}$. We apply Terracini's lemma to find that $S^{k}(X)^{*} \subset X_{k+1}^{*}$. This concludes the proof.

A stronger result than Proposition 1.2.4 has been stated for the first time in [Zak 2004], but no proof was given there.

In the second part of this paper we present a proof of Main Theorem 1.1.5, while in the third part we discuss some consequences and open questions.

\section{Proof of the Main Theorem}

When $Z \subset \mathbb{P}^{N}$, we denote by $\mathscr{C}_{z}(Z) \subset \mathbb{P}^{N}$ the embedded tangent cone to $Z$ at $z$ and if $H \subset \mathbb{P}^{N}$ is a hyperplane, then $[h]$ is the corresponding point in $\left(\mathbb{P}^{N}\right)^{*}$.

The proof of Main Theorem 1.1.5 is obvious if $L^{\perp} \not \subset X^{*}$. Thus, we only deal with the case where $L^{\perp} \subset X^{*}$. Moreover, we can restrict to the case where $X^{*}$ is a hypersurface. Indeed, assume that $X^{*}$ has codimension $p \geq 2$. Let $z \in L^{\perp}$ and $z_{x} \in$ $\left\langle L, T_{X, x}\right\rangle^{\perp}$ be general points, let $M \subset \mathbb{P}^{N}$ be a general $\mathbb{P}^{N+1-p}$ passing through $x$, let $X^{\prime}=M \cap X$ and $L^{\prime}=M \cap L$. We have $\operatorname{Sh}_{X^{\prime}}\left(L^{\prime}\right) \neq X^{\prime}$ and $\left\langle T_{X^{\prime}, x}, L^{\prime}\right\rangle \neq \mathbb{P}^{N+1-p}$. Moreover, we have

$$
\left(X^{\prime}\right)^{*}=\pi_{M^{\perp}}\left(X^{*}\right),
$$

where $\pi_{M^{\perp}}$ is the projection from $M^{\perp}$ in $\mathbb{P}^{N^{*}}$. Since $M$ is general, the map $\pi_{M^{\perp}}$ is locally an isomorphism around $z_{x}$. Hence

$$
\operatorname{mult}_{z} X^{*}=\operatorname{mult}_{z_{x}} X^{*} \Longleftrightarrow \operatorname{mult}_{\pi_{M^{\perp}}(z)}\left(X^{\prime}\right)^{*}=\operatorname{mult}_{\pi_{M^{\perp}}\left(z_{x}\right)}\left(X^{\prime}\right)^{*} .
$$

Finally, note that $\pi_{M^{\perp}}(z)$ is a general point of $\left(L^{\prime}\right)^{\perp}$ and that $\pi_{M^{\perp}}\left(z_{x}\right)$ is a general point of $\left\langle L^{\prime}, T_{X^{\prime}, x}\right\rangle^{\perp}$. As a consequence, it is sufficient to prove the theorem for $X^{\prime}$, whose dual is a hypersurface.

Let's start with a plan of the proof. We assume that $X^{*}$ has constant multiplicity along a smooth curve $S \subset L^{\perp}$ passing through $\left\langle L, T_{X, x}\right\rangle^{\perp}$ and through a general point of $L^{\perp}$ and we find a contradiction. More precisely:

- We prove that the equimultiplicity of $X^{*}$ along $S$ implies that the family of the tangent cones to $X^{*}$ at the points of $S$ is flat.

- Then, we show that the flatness of the family of the tangent cones to $X^{*}$ at the points of $S$ leads to the flatness of the family of the conormal spaces of these tangent cones. As a consequence, we have $\left|\mathscr{C}_{s}\left(X^{*}\right)\right|^{*} \subset L$ for all $s \in S$.

- Finally, we relate the tangent cone to $X^{*}$ at $z$ to the set of tangent hyperplanes to $X^{*}$ at $z$ (when $z$ is a smooth point of $X^{*}$; this is the reflexivity theorem [Kleiman 1986]). Using the fact that $\operatorname{Sh}_{L}(X) \neq X$, we deduce that $\left|\mathscr{C}_{s}\left(X^{*}\right)\right|^{*} \not \subset$ $L$ for $s \in\left\langle L, T_{X, x}\right\rangle^{\perp}$ and thus a contradiction. 
2.1. Normal flatness and Lagrangian specialization principle. Let $S \subset Z \subset \mathbb{P}^{N}$ be two varieties. We recall some properties of the tangent cones $\mathscr{C}_{s}(Z), s \in S$ when $Z$ is equimultiple along $S$.

Definition 2.1.1. Let $S \subset Z$ be two varieties. We say that $Z$ is equimultiple along $S$ if the multiplicity of the local ring $\mathbb{O}_{Z, s}$ is constant for $s \in S$.

Proposition 2.1.2 [Hironaka 1964, Corollary 2, p. 197]. Let $Z \subset \mathbb{P}^{N}$ be a hypersurface and $S$ a connected smooth subvariety (not necessarily closed) of $Z$ such that $Z$ is equimultiple along $S$.

Then, for all $s \in S$, there exists an open neighborhood $U$ of $s$ in $S$ containing $s$ and a closed subscheme $\mathscr{G}(Z) \subset \mathbb{P}^{N} \times U$ such that the natural projection $p:$ $\mathscr{G}(Z) \rightarrow U$ is a flat and surjective morphism whose fiber $\mathscr{G}(Z)_{s^{\prime}}$ over any $s^{\prime} \in U$ is $\mathscr{C}_{s^{\prime}}(Z)$.

We assume that our theorem is not true, that is for general $x \in X$, the multiplicity of $X^{*}$ at a general point of $\left\langle L, T_{X, x}\right\rangle^{\perp}$ is equal to the multiplicity at a general point of $L^{\perp}$.

Let $[h]$ be a general point of $\left\langle L, T_{X, x}\right\rangle^{\perp}$ and let $S \subset L^{\perp}$ be a smooth (not necessarily closed) connected curve passing through $[h]$ and through a general point of $L^{\perp}$. We apply the proposition to $X^{*}$ and $S$. Then there exists a scheme $\mathscr{G}\left(X^{*}\right) \subset \mathbb{P}^{N^{*}} \times S$ such that the natural projection $p: \mathscr{G}\left(X^{*}\right) \rightarrow S$ is a flat and surjective morphism whose fiber over $s \in S$ is the tangent cone to $X^{*}$ at $s$. Let $\Gamma\left(X^{*}\right)=\left|\mathscr{G}\left(X^{*}\right)\right|$. The induced morphism $\Gamma\left(X^{*}\right) \rightarrow S$ is flat and for general $s \in S$ the fiber $\Gamma\left(X^{*}\right)_{s}$ is exactly $\left|\mathscr{C}_{s}\left(X^{*}\right)\right|$.

Now we study the family of the duals of the reduced tangent cones of $X^{*}$ at points of $S$. Applying the Lagrangian specialization principle [Lê and Teissier 1988; Kleiman 1984] to $\Gamma\left(X^{*}\right)$ and $S$, we find:

Theorem 2.1.3. Let $S \subset X^{*}$ be a smooth curve such that $X^{*}$ is equimultiple along $S$. There esists a variety $I_{S}\left(\Gamma\left(X^{*}\right) / \mathbb{P}^{N^{*}} \times S\right)$ with the following properties.

(i) For general $s \in S$, the following equality holds in $\mathbb{P}^{N} \times \Gamma\left(X^{*}\right)_{s}$ :

$$
I\left(\left|\mathscr{C}_{S}\left(X^{*}\right)\right| / \mathbb{P}^{N^{*}}\right)=I_{S}\left(\Gamma\left(X^{*}\right) / \mathbb{P}^{N^{*}} \times S\right)_{S} .
$$

(ii) The morphism $I_{S}\left(\Gamma\left(X^{*}\right) / \mathbb{P}^{N^{*}} \times S\right) \rightarrow S$ is flat and surjective.

(iii) For all $s \in S$, the conormal space $I\left(\left|\mathscr{C}_{s}\left(X^{*}\right)\right| / \mathbb{P}^{N^{*}}\right)$ is a union of irreducible components of the reduced fiber $\left|I_{S}\left(\Gamma\left(X^{*}\right) / \mathbb{P}^{N^{*}} \times S\right)_{s}\right|$.

As a consequence, the image in $\mathbb{P}^{N}$ of the fiber $I_{S}\left(\Gamma\left(X^{*}\right) / \mathbb{P}^{N^{*}} \times S\right)_{s}$, for general $s \in S$, is $\left|\mathscr{C}_{s}\left(X^{*}\right)\right|^{*}$. Moreover, for any $s \in S$, the image of the reduced fiber $\left|I_{S}\left(\Gamma\left(X^{*}\right) / \mathbb{P}^{N^{*}} \times S\right)_{s}\right|$ contains $\left|\mathscr{C}_{S}\left(X^{*}\right)\right|^{*}$. 
2.2. Polar varieties and duals of tangent cones. We discuss an extension of the reflexivity theorem proved in [Lê and Teissier 1988]. The main results of this section will be applied to $X^{*}$ when it is a hypersurface, so we restrict our study to that case.

Definition 2.2.1. Let $Z \subset \mathbb{P}^{N}$ be a reduced and irreducible hypersurface and let $D \subset \mathbb{P}^{N}$ be a linear space. The polar variety of $Z$ associated to $D$, which we denote by $P(Z, D)$, is the closure of the set $\left\{z \in Z_{\text {smooth }}: D \subset T_{Z, z}\right\}$.

If $D=\varnothing$ (that is, $D$ has dimension -1 ), then we put $P(Z, D)=Z$.

Remark 2.2.2. If $Z$ is normal, if $u=\left[u_{0}, \ldots, u_{N}\right]$ in an homogeneous system of coordinates on $\mathbb{P}^{N}$ and $f$ is an equation of $Z$ in this system then $P(Z, u)$ is given by the equations $f=0$ and $u_{0} \partial f / \partial x_{0}+\cdots+u_{N} \partial f / \partial x_{N}=0$.

If $Z$ is not normal, then all irreducible components of $Z_{\text {sing }}$ which are of dimension $N-2$ are irreducible components of the scheme defined by $f=0$ and $u_{0} \partial f / \partial x_{0}+\cdots+u_{N} \partial f / \partial x_{N}=0$, but they are not irreducible components of $P(X, u)$.

Proposition 2.2.3. Let $Z \subset \mathbb{P}^{N}$ be a reduced, irreducible hypersurface and let $D \subset \mathbb{P}^{N}$ be a general linear space of dimension $k$. Then $P(Z, D)$ is empty or of codimension $k+1$ in $Z$.

We state a result of Lê and Teissier which relates the duals of the tangent cones at $z$ of some polar varieties of $Z$ with the tangency locus of $z^{\perp}$ with $Z^{*}$. See [Lê and Teissier 1988, Proposition 2.2.1]. For any $z \in Z$, recall that $\operatorname{Tan}\left(z^{\perp}, Z^{*}\right)$ is the tangency locus of $z$ along $Z^{*}$ (see conormal diagram on page 2 ).

Theorem 2.2.4. Let $Z \subset \mathbb{P}^{N}$ be a reduced and irreducible hypersurface and let $z \in Z$ be a point.

(i) The dual of $\left|\mathscr{C}_{z}(Z)\right|$ is a union of reduced spaces underlying (possibly embedded) components of $\operatorname{Tan}\left(z^{\perp}, Z^{*}\right)$.

(ii) Any irreducible component of $\left|\operatorname{Tan}\left(z^{\perp}, Z^{*}\right)\right|$ is dual to an irreducible component of $\left|\mathscr{C}_{z}(P(Z, D))\right|$ for general $D \in \mathbb{G}(k, N)$ and for some integer $k \in$ $\{-1, \ldots, N-2\}$.

Remark 2.2.5. Part (ii) of the theorem has to be explained. Assume that there is an irreducible component (say $T$ ) of $\left|\operatorname{Tan}\left(z^{\perp}, Z^{*}\right)\right|$ which is not dual to an irreducible component of $\left|\mathscr{C}_{z}(Z)\right|$. Then, there is $k \in\{0, \ldots, N-2\}$ such that for general $D \in \mathbb{G}(k, N)$, we have $z \in P(Z, D)$. Moreover, as $D$ varies in a dense open subset of $\mathbb{G}(k, D)$, the cones $\mathscr{C}_{z}(P(D, Z))$ have a fixed irreducible component in common whose reduced locus is $T^{*}$.

Note also that if $z \in Z_{\text {smooth }}$ then for $k \geq 0$ and for $D$ general in $\mathbb{G}(k, N)$, we have $z \notin P(Z, D)$. As a consequence of the (ii) of the above theorem, we find 
$\operatorname{Tan}\left(z^{\perp}, Z^{*}\right)=T_{Z, z}^{\perp}$ for $z \in Z_{\text {smooth. }}$ This is the way the (obvious corollary of the) reflexivity theorem is often stated.

When $\operatorname{Tan}\left(z^{\perp}, Z^{*}\right)$ is irreducible, one may expect $\left|\mathscr{C}_{z}(Z)\right|^{*}=\left|\operatorname{Tan}\left(z^{\perp}, Z^{*}\right)\right|$. But this is not true:

Example 2.2.6. Let $X \subset \mathbb{P}^{4}$ be the smooth ruled surface of degree 3 considered in example 1.1.1 and let $X^{*}$ its dual. The hypersurface $X^{*}$ has also degree 3 and its singular locus is a $\mathbb{P}^{2}$, the dual of the exceptional section of $X$ (which we denote by $L$ ). Let $C \subset L^{\perp}=X_{\text {sing }}^{*}$ be the conic corresponding to the hyperplanes which are tangent to $X$ along a ruling of $X$ and let $z \in C$.

The tangent cone $\mathscr{C}_{z}\left(X^{*}\right)$ is a doubled $\mathbb{P}^{3}$ so that $\left|\mathscr{C}_{z}\left(X^{*}\right)\right|^{*} \neq \operatorname{Tan}\left(z^{\perp}, X\right)$. We also note that the scheme-theoretic tangency locus of $z^{\perp}$ along $X$ is a line with an embedded point. The embedded point is dual to $\left|\mathscr{C}_{z}\left(X^{*}\right)\right|$ and the line is dual to $\left|\mathscr{C}_{z}\left(P\left(X^{*}, u\right)\right)\right|$, for general $u \in \mathbb{P}^{4 *}$.

Notations 2.2.7. Let $f: Y \rightarrow T$ be a quasiprojective morphism between quasiprojective schemes, let $T^{\prime} \subset T$ be a smooth variety and let $s \in T^{\prime}$ be any point. Let $Y_{1}, \ldots, Y_{m}$ be the irreducible components of $f^{-1}\left(T^{\prime}\right)$ such that the restrictions

$$
\left.f\right|_{Y_{i}}: Y_{i} \rightarrow T^{\prime},
$$

are surjective. Define the scheme

$$
\operatorname{limflat}_{\left\{t \rightarrow s, t \in T^{\prime}\right\}} f^{-1}(t):=\left.f\right|_{Y_{1} \cup \cdots \cup Y_{m}} ^{-1}(s) .
$$

If $\operatorname{dim}\left(T^{\prime}\right)=1$ and the $Y_{i}$ are all reduced, this is the classical flat limit taken along a smooth curve. If $\left.f\right|_{f^{-1}\left(T^{\prime}\right)}: f^{-1}\left(T^{\prime}\right) \rightarrow T^{\prime}$ is flat, then

$$
\operatorname{limflat}_{\left\{t \rightarrow s, t \in T^{\prime}\right\}} f^{-1}(t)=\left.f\right|_{f^{-1}\left(T^{\prime}\right)} ^{-1}(s) .
$$

Proof of Main Theorem 1.1.5. We recall the setting for the convenience of the reader. The projective variety $X \subset \mathbb{P}^{N}$ is irreducible and nondegenerate. The linear space $L \subset \mathbb{P}^{N}$ is such that $\operatorname{Sh}_{X}(L) \neq X$ and $\left\langle L, T_{X, x}\right\rangle \neq \mathbb{P}^{N}$ for all $x \in X_{\text {smooth }}$. We want to prove that for all $x \in X_{\text {smooth }}$ such that $x \notin \operatorname{Sh}_{X}(L)$, the multiplicity in $X^{*}$ of a general hyperplane containing $\left\langle L, T_{X, x}\right\rangle$ is strictly greater than that of a general hyperplane containing $L$.

The result is obvious if $L^{\perp} \not \subset X^{*}$ and we have already seen that we can restrict to the case where $X^{*}$ is a hypersurface. So we only consider the case where $L^{\perp} \subset X^{*}$ and $X^{*}$ is a hypersurface and we assume that our result is not true. Let $x \in X_{\text {smooth }}$ with $x \notin \operatorname{Sh}_{X}(L)$ and let $[h]$ be a general point in $\left\langle L, T_{X, x}\right\rangle^{\perp}$. By the results of the previous section, there exists a smooth (not necessarily closed) curve $S \subset L^{\perp}$ with $[h] \in S$ and a flat morphism

$$
I_{S}\left(\Gamma\left(X^{*}\right) / \mathbb{P}^{N^{*}} \times S\right) \rightarrow S,
$$


whose fiber $I_{S}\left(\Gamma\left(X^{*}\right) / \mathbb{P}^{N^{*}} \times S\right)_{s}$ is the conormal space of $\left|\mathscr{C}_{S}\left(X^{*}\right)\right|$, for general $s \in S$. Further, the conormal space of $\left|\mathscr{C}_{S}\left(X^{*}\right)\right|$ is included in $\left|I_{S}\left(\Gamma\left(X^{*}\right) / \mathbb{P}^{N^{*}} \times S\right)_{s}\right|$ for all $s \in S$.

Theorem 2.2.4(i) implies that

$$
\left|\mathscr{C}_{s}\left(X^{*}\right)\right|^{*} \subset p\left(\left|q^{-1}(s)\right|\right),
$$

for all $s \in S$, where $p$ and $q$ are as in the conormal diagram of page 2. The flatness of $I_{S}\left(\Gamma\left(X^{*}\right) / \mathbb{P}^{N^{*}} \times S\right) \rightarrow S$ gives the inclusion

$$
\left|\mathscr{C}_{[h]}\left(X^{*}\right)\right|^{*} \subset p\left(\operatorname{limflat}_{\{s \rightarrow[h], s \in S\}}\left|q^{-1}(s)\right|\right) .
$$

By Definition 1.1.2, the right-hand side is contained in $\operatorname{Tan}(L, X) \subset L$.

Let $\mathscr{F}$ be an irreducible component of $\operatorname{Tan}(H, X)$ passing through $x$. By Theorem 2.2.4, there is an integer $k \in\{-1, \ldots, N-2\}$ such that $|\mathscr{F}|$ is dual to an irreducible component of $\left|\mathscr{C}_{[h]}\left(P\left(X^{*}, D\right)\right)\right|$, for general $D \in \mathbb{G}(k, N)$. Since $\left|\mathscr{C}_{[h]}\left(X^{*}\right)\right|^{*} \subset L$, we have $k \geq 0$.

Let $x_{0} \in \mathscr{F}$ be a general point. Duality implies $T_{\left|\mathscr{C}_{[h]}\left(P\left(X^{*}, D\right)\right)\right|, z} \subset x_{0}^{\perp}$ for some general $z$ in the irreducible component of $\mathscr{C}_{[h]}\left(P\left(X^{*}, D\right)\right)$ whose reduced locus is $|\mathscr{F}|^{*}$. Note that $\mathscr{C}_{[h]}\left(P\left(X^{*}, D\right)\right) \subset \mathscr{C}_{[h]}\left(X^{*}\right)$. Let $T_{\left[\mathscr{C}_{[h]}\left(X^{*}\right) \mid, z\right.}$ be a limit of tangent spaces to $\left|\mathscr{C}_{[h]}\left(X^{*}\right)\right|$ at $z$. The point $z$ is general in $\left|\mathscr{C}_{[h]}\left(P\left(X^{*}, D\right)\right)\right|$, so $T_{\left|\mathscr{C}_{[h]}\left(P\left(X^{*}, D\right)\right)\right|, z} \subset T_{\left|\mathscr{C}_{[h]}\left(X^{*}\right)\right|, z}$.

As a consequence of this, we have $T_{\left|\mathscr{C}_{[h]}\left(P\left(X^{*}, D\right)\right)\right|, z} \subset x_{0}^{\perp} \cap T_{\mathscr{C}_{[h]}\left(X^{*}\right), z}$. That is,

$$
\left\langle x_{0}, T_{\left|\mathscr{C}_{[h]}\left(X^{*}\right)\right|, z}^{\perp}\right\rangle \subset \mathscr{F} \subset X .
$$

But $\left|\mathscr{C}_{[h]}\left(X^{*}\right)\right|^{*} \subset \operatorname{Tan}(L, X)$, so $T_{\left|\mathscr{C}_{[h]}\left(X^{*}\right)\right|, z}^{\perp} \in \operatorname{Tan}(L, X)$, and the inclusion above says that $x_{0} \in \mathrm{Sh}_{X}(L)$. This is a contradiction.

\section{Corollaries and open questions}

We present here some corollaries of the Main Theorem and related open questions.

3.1. Zak's conjecture on varieties with minimal codegree. Let $X \subset \mathbb{P}^{N}$ be an irreducible, nondegenerate projective variety. We recall, following Zak, that the order of $X$ is ord $X=\min \left\{k, S^{k-1}(X)=\mathbb{P}^{N}\right\}$ and the $k$-th secant-defect is $\delta_{k}=$ $\operatorname{dim} X+\operatorname{dim} S^{k-1}(X)+1-\operatorname{dim} S^{k}(X)$, for all $k \leq \operatorname{ord} X-1$.

Zak [1993] proved an important result related to secant defects.

Theorem 3.1.1 (Zak's superadditivity theorem). Let $X \subset \mathbb{P}^{N}$ an irreducible, nondegenerate projective variety such that $\delta_{1}>0$. For all $k \leq \operatorname{ord} X-1$, we have the inequality

$$
\delta_{k} \geq \delta_{k-1}+\delta_{1} .
$$

The varieties on the boundary are called Scorza varieties. More precisely: 
Definition 3.1.2. An irreducible, smooth, nondegenerate projective variety $X \subset$ $\mathbb{P}^{N}$ is a Scorza variety if the following conditions hold:

(i) $\delta_{1}>0$ and $N>2 n+1-\delta_{1}$,

(ii) $\delta_{k}=\delta_{k-1}+\delta_{1}$ for all $k \leq$ ord $X-1$,

(iii) $\operatorname{ord} X-1=\left[\operatorname{dim} X / \delta_{1}\right]$, where [ ] denotes the integral part.

Theorem 3.1.3 (Classification of Scorza varieties [Zak 1993]). Any Scorza variety $X$ is of one of the following types:

(i) $X=v_{2}\left(\mathbb{P}^{n}\right) \subset \mathbb{P}^{n(n+3) / 2}\left(2^{\text {nd }}\right.$ Veronese) and $\operatorname{deg} X^{*}=n+1$;

(ii) $X=\mathbb{P}^{n} \times \mathbb{P}^{n} \subset \mathbb{P}^{n(n+2)}$ and $\operatorname{deg} X^{*}=n+1$;

(iii) $X=\mathbb{G}(1,2 n+1) \subset \mathbb{P}\left(\Lambda^{2} \mathbb{C}^{2 n+2}\right)$ and $\operatorname{deg}\left(X^{*}\right)=n+1$;

(iv) $X \subset \mathbb{P}^{26}$ is the 16-dimensional variety corresponding to the orbit of highest weight vector in the lowest nontrivial representation of the group of type $E_{6}$ and $\operatorname{deg} X^{*}=3$.

In [Zak 2004] an important consequence of the assertion $S^{k}(X)^{*} \subset X_{k+1}^{*}$ (where $X_{k}^{*}$ is the set of points of multiplicity at least $k$ in $X^{*}$ ) was discovered. We state that result in the setting where we are able to prove it.

Proposition 3.1.4. Let $X \subset \mathbb{P}^{N}$ be an irreducible, nondegenerate, smooth, projective variety. Assume that $X$ is not $k$ dual defective for $k<\operatorname{ord} X-1$, then

$$
\operatorname{deg} X^{*} \geq \operatorname{ord} X
$$

Proof. With the assumptions above, Proposition 1.2.4 implies that there is a point of multiplicity ord $X-1$ in $X^{*}$. Since $X$ is nondegenerate, its dual is not a cone and so $\operatorname{deg} X^{*} \geq \operatorname{ord} X$.

If $X$ is a Scorza variety then $\operatorname{deg} X^{*}=\operatorname{ord} X$. The converse statement in conjectured in [Zak 2004]. We formulate the conjecture in the setting where we can prove the inequality: $\operatorname{deg} X^{*} \geq \operatorname{ord} X$.

Conjecture 3.1.5 [Zak 2004]. Let $X \subset \mathbb{P}^{N}$ be an irreducible, smooth, nondegenerate, projective variety. Assume that $X$ is not $k$ dual defective for all $k<\operatorname{ord} X$ and that $\operatorname{deg} X^{*}=\operatorname{ord} X+1$, then $X$ is a hyperquadric or a Scorza variety.

It is proved in [Zak 1993], without any hypothesis on the dual defectiveness of $X$, that smooth varieties with $\operatorname{deg}\left(X^{*}\right)=3$ and ord $X=3$ are Severi varieties. In particular, they are Scorza varieties. Note, however, that the smoothness assumption seems to be necessary in his proof. I believe it would be very interesting to have a classification of all varieties whose duals have degree 3 . 
3.2. Varieties with unexpected equisingular linear spaces. We come back to our usual setting. Let $L \subset \mathbb{P}^{N}$ be a linear space such that for all $x \in X_{\text {smooth }}$, we have $\left\langle L, T_{X, x}\right\rangle \neq \mathbb{P}^{N}$. We have seen in example 1.1.1 that a hyperplane containing the join $\left\langle L, T_{X, x}\right\rangle$ may have the same multiplicity in $X^{*}$ as the general hyperplane containing $L$, even if $x$ is a general point of $X$. The following definition is convenient to describe this situation.

Definition 3.2.1. Let $X \subset \mathbb{P}^{N}$ be an irreducible, nondegenerate projective variety such that $X^{*}$ is a hypersurface. Let $L \subset \mathbb{P}^{N}$ be a linear space such that for all $x \in X_{\text {smooth }}$, we have $\left\langle L, T_{X, x}\right\rangle \neq \mathbb{P}^{N}$. We say that $L^{\perp}$ is an unexpected equisingular linear space in $X^{*}$ if for all $x \in X_{\text {smooth }}$, the general hyperplane containing $\left\langle L, T_{X, x}\right\rangle$ has the same multiplicity in $X^{*}$ as the general hyperplane containing $L$.

The variety in Example 1.1.1 is rather special since it is a scroll surface (see [Zak 2004] for interesting discussions about this variety). It is not a coincidence that the directrix of this variety is an unexpected equisingular linear space in its dual. Indeed, we have:

Theorem 3.2.2. Let $X \subset \mathbb{P}^{N}$ be an irreducible, smooth, nondegenerate projective variety such that $X^{*}$ is a hypersurface. Let $L \subset X$ be a linear space with $\operatorname{dim}(L)=$ $\operatorname{dim}(X)-1$. Assume that $L^{\perp}$ is an unexpected equisingular linear space in $X^{*}$ such that mult $L_{L^{\perp}} X^{*}=2$. Then $X$ is the cubic scroll surface in $\mathbb{P}^{4}$.

Here mult $L_{L^{\perp}} X^{*}$ denotes the multiplicity in $X^{*}$ of a general point of $L^{\perp}$. Before diving into the proof of Theorem 3.2.2, we describe the tangency locus of any point $[h] \subset X^{*}$, such that mult $[h] X^{*}=2$.

Proposition 3.2.3. Let $X \subset \mathbb{P}^{N}$ be a smooth, irreducible, nondegenerate projective variety such that $X^{*}$ is a hypersurface. Let $[h] \in X^{*}$ be such that mult $_{[h]} X^{*}=2$. The scheme theoretic tangency locus of $H$ with $X$ is either

(i) an irreducible hyperquadric and in this case $\left|\mathscr{C}_{[h]}\left(X^{*}\right)\right|^{*}=\operatorname{Tan}(H, X)$,

(ii) the union of two (not necessarily distinct) linear spaces, or

(iii) a linear space with at least one embedded component.

We postpone the proof of this result to the Appendix.

Proof of Theorem 3.2.2. Let $H$ be a general hyperplane containing $L$. We have $H \cap X=L \cup D_{H}$, where $D_{H}$ is a divisor such that

$$
D_{H} \cap L=\operatorname{Tan}(H, X) .
$$

Let $x \in X$ be a general point and let $H_{x}$ be a general hyperplane containing $\left\langle L, T_{X, x}\right\rangle$. Then $\operatorname{Tan}\left(H_{x}, X\right)$ contains $x$ and

$$
\xi:=p\left(\operatorname{limflat}_{\left\{[h] \rightarrow\left[h_{x}\right],[h] \in L^{\perp}\right\}} q^{-1}([h])\right) .
$$


By hypothesis, we have

$$
\operatorname{mult}_{\left[h_{x}\right]} X^{*}=\operatorname{mult}_{[h]} X^{*}=2,
$$

for all $[h] \in L^{\perp}$. Proposition 3.2.3 hence implies that the irreducible component of $\operatorname{Tan}\left(H_{x}, X\right)$ containing $x$, which we denote by $R_{H_{x}}$, also contains $\xi$. Moreover, $\xi \subset L$, so $\operatorname{dim} R_{H_{x}}>\operatorname{dim} \xi$, for general $[h] \in L^{\perp}$. As a consequence, $\operatorname{dim} R_{H_{x}}=$ $n-1$.

On the other hand, since

$$
\operatorname{mult}_{\left[h_{x}\right]} X^{*}=\operatorname{mult}_{[h]} X^{*}=2,
$$

for all $[h] \in L^{\perp}$, we have $\left|\mathscr{C}_{\left[h_{x}\right]}\left(X^{*}\right)\right|^{*} \neq\left|R_{H_{x}}\right|$. We apply again Proposition 3.2.3 and we find that $\left|R_{H_{x}}\right|$ is necessarily a linear space of dimension $n-1$. Thus,

$$
\operatorname{dim}\left\langle L, T_{X, x}\right\rangle=n+1 .
$$

Note that Bertini's theorem implies that

$$
R_{H_{x}} \subset\left\langle L, T_{X, x}\right\rangle \cap X,
$$

for general $H_{x}$ containing $\left\langle L, T_{X, x}\right\rangle$. As a consequence $R_{H_{x}}$ is an irreducible component of $\left\langle L, T_{X, x}\right\rangle \cap X$, for general $H_{x}$. Thus $R_{H_{x}}$ does not depend on $H_{x}$, for general $H_{x}$ containing $\left\langle L, T_{X, x}\right\rangle$. We deduce that $\left\langle L, T_{X, x}\right\rangle$ is tangent to $X$ along a linear space of dimension $n-1$. By the theorem on tangencies, we have $n-1 \leq 1$, that is $n=2$ (obviously, $X$ is not a curve). So $X \subset \mathbb{P}^{N}$ is a nondegenerate surface containing a distinguished line $L$, such that for general $x \in X$, there is a $\mathbb{P}^{3}$ tangent to $X$ along a line passing through $x$ and meeting $L$. This means that $X$ is the projection of a scroll of type $S_{1, d-1}$. By hypothesis, we have mult $L_{L^{\perp}} X^{*}=2$, hence of [Ciliberto et al. 2008, Proposition 1.6] implies that $X=S_{1,2} \subset \mathbb{P}^{4}$.

\section{Appendix: Tangency loci of points of multiplicity 2 in the dual}

The goal of this appendix is to prove the following proposition.

Proposition 3.2.3. Let $X \subset \mathbb{P}^{N}$ be a smooth, irreducible, nondegenerate projective variety such that $X^{*}$ is a hypersurface. Let $[h] \in X^{*}$ be such that mult $[h] X^{*}=2$. The scheme theoretic tangency locus of $H$ with $X$ is either

(i) an irreducible hyperquadric and in this case $\left|\mathscr{C}_{[h]}\left(X^{*}\right)\right|^{*}=\operatorname{Tan}(H, X)$,

(ii) the union of two (not necessarily distinct) linear spaces, or

(iii) a linear space with at least one embedded component.

Example A.1. All three cases are encountered in nature:

(i) If $X=v_{2}\left(\mathbb{P}^{2}\right) \subset \mathbb{P}^{5}$, then for all $[h] \in v_{2}\left(\mathbb{P}^{2^{*}}\right) \subset X^{*}$, we have mult $[h] X^{*}=2$ and $\operatorname{Tan}(H, X)$ is a smooth conic. 
(ii) If $X$ is a complete intersection of large multidegree and large codimension, then there are points $\left[h_{1}\right],\left[h_{2}\right] \in X^{*}$ such that $\operatorname{mult}_{\left[h_{i}\right]} X^{*}=2$ and $\operatorname{Tan}\left(H_{1}, X\right)$ is exactly two distinct points, whereas $\operatorname{Tan}\left(H_{2}, X\right)$ is a single double point.

(iii) If $X$ is the cubic scroll of Example 1.1.1, then there is a conic $C \subset X^{*}$, such that for all $[h] \in C$, we have mult $[h] X^{*}=2$ and $\operatorname{Tan}(H, X)$ is a line with an embedded point.

A doubled linear space will be considered as the union of two (not distinct) linear spaces. By Theorem 2.2.4, we know that the irreducible components of $\operatorname{Tan}(H, X)$ are dual to irreducible components of the reduced spaces underlying some $\mathscr{C}_{[h]}\left(P\left(X^{*}, D_{k}\right)\right)$ for general $D_{k} \in \mathbb{G}(k, N)$. When mult $[h] X^{*}=2$, the cones $\mathscr{C}_{[h]}\left(P\left(X^{*}, D_{k}\right)\right)$ are rather easy to describe. Let's start with some notation.

Notations A.2. Let $Z \subset \mathbb{P}^{N}$ be a reduced and irreducible hypersurface. Let $D \in$ $\mathbb{G}(k, N)$ and let $f_{Z}$ be an equation for $Z$ in some coordinate system of $\mathbb{P}^{N}$. We denote by $P\left(f_{Z}, D\right)$ the subscheme of $\mathbb{P}^{N}$ whose ideal is generated by the equations

$$
u_{0} \frac{\partial f_{Z}}{\partial t_{0}}+\cdots+u_{N} \frac{\partial f_{Z}}{\partial t_{N}}
$$

for $u=\left[u_{0}, \ldots, u_{N}\right]$ varying in $D$.

Let $D \in \mathbb{G}(k, N)$ be a general $k$-plane. Note that if $\operatorname{dim}\left(Z_{\text {sing }}\right)<\operatorname{dim} P(Z, D)$ (that is $\left.\operatorname{dim} Z_{\text {sing }} \leq N-k-3\right)$, then $P(Z, D)=P\left(f_{Z}, D\right) \cap Z$. In the other case, the irreducible components of maximal dimension of $Z_{\text {sing }}$ are irreducible components of $P\left(f_{Z}, D\right) \cap Z$.

Lemma A.3. Let $Z \subset \mathbb{P}^{N}$ be an irreducible and reduced hypersurface. Let $z \in Z$ and let $k \in\{-1, \ldots, N-2\}$. Then, for general $D \in \mathbb{G}(k, N)$, we have

(1) $z \notin P(Z, D)$, or

(2) $\operatorname{mult}_{z} P(Z, D)=\operatorname{mult}_{z}(Z)$. mult $z\left(f_{Z}, D\right)$, if $\operatorname{dim}\left(Z_{\text {sing }}^{(z)}\right)<\operatorname{dim} P(Z, D)$, where $Z_{\text {sing }}^{(z)}$ is an irreducible component of $Z_{\text {sing }}$ of maximal dimension passing through $z$, or

(3) $\operatorname{mult}_{z} P(Z, D)<\operatorname{mult}_{z}(Z)$. mult $z\left(f_{Z}, D\right)$, if $\operatorname{dim}\left(Z_{\text {sing }}^{(z)}\right) \geq \operatorname{dim} P(Z, D)$, where $Z_{\text {sing }}^{(z)}$ is an irreducible component of $Z_{\text {sing }}$ of maximal dimension passing through $z$.

Proof. If $z \in P(Z, D)$ for general $D \in \mathbb{G}(k, N)$, we will prove the lemma only in the case $P\left(f_{Z}, D\right)$ is smooth at $z$, for two reasons. The general case is obtained by the same methods, this is only more technical, and we will use the result only in the case $P\left(f_{Z}, D\right)$ is smooth at $z$.

Moreover if $z \in P(Z, D)$ for general $D$, we will only concentrate on the case $\operatorname{dim}\left(Z_{\text {sing }}^{(s)}\right)<\operatorname{dim} P(Z, D)$. In this case, we have locally around $z$ the equality 
$P(Z, D)=P\left(f_{Z}, D\right) \cap Z$ for general $D \in \mathbb{G}(k, N)$. The situation where an irreducible component $Z_{\text {sing }}$ containing $z$ is an irreducible component of $P\left(f_{Z}, D\right) \cap$ $Z$ - this is case (3) of the lemma - is dealt with exactly in the same way.

Now, we work locally around $z$, so that $P\left(f_{Z}, D\right) \cap Z=P(Z, D) \subset \mathbb{A}^{N}$, for general $D \in \mathbb{G}(k, N)$. Let $\left(Z_{i}\right)_{i \in I}$ be a stratification of $Z$ such that $Z_{i}$ is smooth and $Z$ is normally flat along $Z_{i}$, for all $i \in I$. Such a stratification exists, due to the open nature of normal flatness (see [Hironaka 1964, Chapter II]). Consider the Gauss map $G: Z \rightarrow \mathbb{P}^{N^{*}}$. It restricts to a map $G_{i}: Z_{i} \rightarrow \mathbb{P}^{N^{*}}$. We have

$$
P\left(f_{Z}, D\right) \cap Z=P(Z, D)=G^{-1}\left(D^{\perp}\right),
$$

so that $P\left(f_{Z}, D\right) \cap Z_{i}=G_{i}^{-1}\left(D^{\perp}\right)$, for all $i$.

Now, we apply Kleiman's transversality theorem to find that for all $i$ and for general $D \in \mathbb{G}(k, N)$, the inverse images $G_{i}^{-1}\left(D^{\perp}\right)$ are either empty or smooth of the expected dimension.

Let $i$ such that $z$ is in $Z_{i}$. If $z \notin G_{i}^{-1}\left(D^{\perp}\right)$ for general $D \in \mathbb{G}(k, N)$, then $z \notin P(Z, D)$ and we are in the case 1 of the lemma. Otherwise, $z$ is a smooth point of $G_{i}^{-1}\left(D^{\perp}\right)$, so $T_{P\left(f_{Z}, D\right), z}$ and $T_{Z_{i}, z}$ are transverse.

Assume that mult $P(Z, D)>$ mult $_{z} Z$. mult $P\left(f_{Z}, D\right)$. Since $P\left(f_{Z}, D\right)$ is smooth at $z$, this implies that $T_{P\left(f_{Z}, D\right), z}$ and $\mathscr{C}_{Z}(Z)$ are not transverse. In particular, the linear spaces $T_{P\left(f_{Z}, D\right), z}$ and $\operatorname{Vert}\left(\mathscr{C}_{z}(Z)\right)$ are not transverse (here $\operatorname{Vert}\left(\mathscr{C}_{z}(Z)\right)$ is the vertex of the cone $\left.\mathscr{C}_{z}(Z)\right)$. But $Z$ is normally flat along $Z_{i}$, so we have $T_{Z_{i}, z} \subset \operatorname{Vert}\left(\mathscr{C}_{Z}(Z)\right)$ (see [Hironaka 1964, Theorem 2, p. 195]). This is a contradiction.

Corollary A.4. Let $Z \subset \mathbb{P}^{N}$ be a reduced, irreducible hypersurface. Let $z \in Z$ such that mult $_{z} Z=2$ and let $k \in\{-1, \ldots, N-2\}$. Then, for general $D \in \mathbb{G}(k, N)$, we have

$$
\operatorname{mult}_{z} P(Z, D) \leq 2 \text {. }
$$

Proof. The result is obvious for $k=-1$, since in this case $P(Z, D)=Z$. Assume that $k \geq 0$ and let $D \in \mathbb{G}(k, N)$ be a general $k$-plane. Let $u \in D$ be a general point ans let $\pi_{u}$ be the projection from $u$. Then, the projections

$$
\left.\pi_{u}\right|_{P(Z, u)}: P(Z, u) \rightarrow \pi_{u}(P(Z, u))
$$

and

$$
\left.\pi_{u}\right|_{P(Z, D)}: P(Z, D) \rightarrow \pi_{u}(P(Z, D))
$$

are locally isomorphisms around $z$. Moreover, we have the following equality (see [Teissier 1982]):

$$
\pi_{u}(P(Z, D))=P\left(\pi_{u}(P(Z, u)), \pi_{u}(D)\right) .
$$


As a consequence, it is sufficient to prove the result for $k=0$. But in this case, this is an obvious application of the lemma above. Indeed, for general $u \in \mathbb{P}^{N}$,

$$
\operatorname{mult}_{z} P\left(f_{Z}, u\right)=\text { mult }_{z} Z-1=1 .
$$

We also need the following result.

Proposition A.5. Let $X \subset \mathbb{P}^{N}$ be an irreducible projective variety such that $X^{*}$ is a hypersurface. Let $[h] \in X^{*}$ be such that $\operatorname{Tan}(H, X)$ has $m$ components (some of which may be embedded components), then there exists $k \in\{-1, \ldots, N-2\}$, such that for general $D \in G(k, N)$, we have

$$
\operatorname{mult}_{[h]} P\left(X^{*}, D\right) \geq m .
$$

Proof. We only prove the result when $\operatorname{Tan}(H, X)$ is reduced and pure dimensional. The general case is done using the same ideas; it's just more technical.

Assume that

$$
\operatorname{Tan}(H, X)=Y_{1} \cup \cdots \cup Y_{m},
$$

where the $Y_{i}$ have the same codimension, say $c$. Let $D \subset \mathbb{P}^{N^{*}}$ be a general $\mathbb{P}^{N-1-c}$. Then

$$
\pi_{D}\left(P\left(X^{*}, D\right)\right)=\left(D^{\perp} \cap X\right)^{*},
$$

where $\pi_{D}$ is the projection from $D$. Moreover, we have $[h] \in P\left(X^{*}, D\right)$ and

$$
\operatorname{Tan}\left(D^{\perp} \cap H, D^{\perp} \cap X\right)=D^{\perp} \cap \operatorname{Tan}(H, X) .
$$

As a consequence, $\operatorname{Tan}\left(D^{\perp} \cap H, D^{\perp} \cap X\right)$ is a 0-dimensional scheme of degree at least $m$. In this case, it is clear that

$$
\operatorname{mult}_{\pi_{D}([h])} \pi_{D}\left(P\left(X^{*}, D\right)\right) \geq m .
$$

On the other hand, since $D$ is general, the morphism

$$
\pi_{D}: P\left(X^{*}, D\right) \rightarrow \pi_{D}\left(P\left(X^{*}, D\right)\right)
$$

is locally an isomorphism around $[h]$, so that

$$
\operatorname{mult}_{[h]} P\left(X^{*}, D\right) \geq m .
$$

Proof of Proposition 3.2.3. Let $T_{1} \cup \cdots \cup T_{m}$ be the decomposition of $\operatorname{Tan}(H, X)$ into irreducible components. If $m \geq 3$, then Proposition A.5 implies that $\operatorname{mult}_{[h]}\left(X^{*}\right) \geq$ 3 , this is impossible, so that $m \leq 2$.

Assume that $m=2$. The proof of Proposition A.5 shows that these two irreducible components are scheme-theoretically linear spaces.

Assume that $m=1$ and let $k \in\{-1, \ldots, N-2\}$ such that $T_{1}$ is dual to some irreducible components of the reduced space underlying $\mathscr{C}_{[h]} P\left(X^{*}, D\right)$, for general $D \in \mathbb{G}(k, N)$. By Corollary A.4, the cone $\mathscr{C}_{[h]} P\left(X^{*}, D\right)$ is either a hyperquadric 
or a linear space. Assume that it is an irreducible hyperquadric. If $k \geq 0$, we know by Theorem 2.2.4 that $\left|\mathscr{C}_{[h]}\left(X^{*}\right)\right|^{*}$ is the reduced space underlying some embedded component of $\operatorname{Tan}(H, X)$. Taking $q=\operatorname{dim} \operatorname{Tan}(H, X)$ general hyperplane sections of $\operatorname{Tan}(H, X)$ passing through $\left|\mathscr{C}_{[h]}\left(X^{*}\right)\right|^{*}$, we see as in the proof of Proposition A.5 that for general $D^{\prime} \in \mathbb{G}(q-1, N)$, we have

$$
\operatorname{mult}_{[h]} P\left(X^{*}, D^{\prime}\right) \geq 3 \text {. }
$$

This is impossible by Corollary A.4. Thus, if $\mathscr{C}_{[h]} P\left(X^{*}, D\right)$ is an irreducible hyperquadric, then $k=-1$, and we are in the case 1 of the proposition.

Finally, if $\mathscr{C}_{[h]} P\left(X^{*}, D\right)$ is a the union of two linear spaces or a unique linear space, then we are in case 2 or 3 of the proposition. This concludes the proof of Proposition 3.2.3.

\section{Acknowledgements}

I thank Bernard Teissier who took time to explain me part of his work and Christine Jost for interesting discussions on Segre classes. I am also especially grateful to Christian Peskine for the numerous discussions we had together. This work owes a lot to his patience. Finally, my thanks go to the referee, who helped me to improve the paper, in many ways.

\section{References}

[Chiantini and Ciliberto 2010] L. Chiantini and C. Ciliberto, "On the dimension of secant varieties", J. Eur. Math. Soc. 12:5 (2010), 1267-1291. MR 2011m:14088 Zbl 1201.14038

[Ciliberto et al. 2008] C. Ciliberto, F. Russo, and A. Simis, "Homaloidal hypersurfaces and hypersurfaces with vanishing Hessian”, Adv. Math. 218:6 (2008), 1759-1805. MR 2009j:14056 Zbl 1144.14009

[Hironaka 1964] H. Hironaka, "Resolution of singularities of an algebraic variety over a field of characteristic zero, I", Ann. of Math. (2) 79 (1964), 109-203. MR 33 \#7333

[Kleiman 1984] S. L. Kleiman, "About the conormal scheme", pp. 161-197 in Complete intersections (Acireale, 1983), edited by S. Greco and R. Strano, Lecture Notes in Math. 1092, Springer, Berlin, 1984. MR 87g:14060 Zbl 0547.14031

[Kleiman 1986] S. L. Kleiman, "Tangency and duality”, pp. 163-225 in Proceedings of the 1984 Vancouver conference in algebraic geometry, edited by J. Carrell et al., CMS Conf. Proc. 6, Amer. Math. Soc., Providence, RI, 1986. MR 87i:14046 Zbl 0601.14046

[Lê and Teissier 1988] Lê D. T. and B. Teissier, "Limites d'espaces tangents en géométrie analytique”, Comment. Math. Helv. 63:4 (1988), 540-578. MR 89m:32025 Zbl 0658.32010

[Teissier 1982] B. Teissier, "Variétés polaires, II: multiplicités polaires, sections planes, et conditions de Whitney”, pp. 314-491 in Algebraic geometry (La Rábida, 1981), edited by J. M. Aroca et al., Lecture Notes in Math. 961, Springer, Berlin, 1982. MR 85i:32019 Zbl 0585.14008

[Zak 1993] F. L. Zak, Tangents and secants of algebraic varieties, Transl. Math. Monographs 127, American Mathematical Society, Providence, RI, 1993. MR 94i:14053 Zbl 0795.14018 
[Zak 2004] F. L. Zak, "Determinants of projective varieties and their degrees", pp. 207-238 in Algebraic transformation groups and algebraic varieties, edited by V. L. Popov, Encyclopaedia Math. Sci. 132, Springer, Berlin, 2004. MR 2005h:14128 Zbl 1063.14058

Received July 20, 2010. Revised March 10, 2011.

ROLAND ABUAF

INSTITUT FOURIER

100 RUE DES MATHS, BP 74

38402 SAINT-MARTIN D'HÈRES

FRANCE

abuaf@ujf-grenoble.fr 


\title{
PACIFIC JOURNAL OF MATHEMATICS
}

\author{
http://www.pjmath.org \\ Founded in 1951 by
}

E. F. Beckenbach (1906-1982) and F. Wolf (1904-1989)

\section{EDITORS}

V. S. Varadarajan (Managing Editor)

Department of Mathematics

University of California

Los Angeles, CA 90095-1555

pacific@math.ucla.edu

Vyjayanthi Chari

Department of Mathematics

University of California

Riverside, CA 92521-0135

chari@math.ucr.edu

\section{Robert Finn}

Department of Mathematics Stanford University

Stanford, CA 94305-2125

finn@math.stanford.edu

Kefeng Liu

Department of Mathematics

University of California

Los Angeles, CA 90095-1555

liu@math.ucla.edu
Darren Long

Department of Mathematics

University of California

Santa Barbara, CA 93106-3080

long@math.ucsb.edu

Jiang-Hua Lu

Department of Mathematics

The University of Hong Kong

Pokfulam Rd., Hong Kong jhlu@maths.hku.hk

Alexander Merkurjev

Department of Mathematics University of California

Los Angeles, CA 90095-1555 merkurev@math.ucla.edu
Sorin Popa

Department of Mathematics

University of California

Los Angeles, CA 90095-1555

popa@math.ucla.edu

Jie Qing

Department of Mathematics

University of California

Santa Cruz, CA 95064

qing@ cats.ucsc.edu

Jonathan Rogawski

Department of Mathematics

University of California

Los Angeles, CA 90095-1555

jonr@math.ucla.edu

\section{PRODUCTION}

pacific@math.berkeley.edu

Silvio Levy, Scientific Editor Matthew Cargo, Senior Production Editor

ACADEMIA SINICA, TAIPEI

CALIFORNIA INST. OF TECHNOLOGY

INST. DE MATEMÁTICA PURA E APLICADA

KEIO UNIVERSITY

MATH. SCIENCES RESEARCH INSTITUTE

NEW MEXICO STATE UNIV.

OREGON STATE UNIV.

\section{SUPPORTING INSTITUTIONS}

STANFORD UNIVERSITY
UNIV. OF BRITISH COLUMBIA
UNIV. OF CALIFORNIA, BERKELEY
UNIV. OF CALIFORNIA, DAVIS
UNIV. OF CALIFORNIA, LOS ANGELES
UNIV. OF CALIFORNIA, RIVERSIDE
UNIV. OF CALIFORNIA, SAN DIEGO
UNIV. OF CALIF., SANTA BARBARA

UNIV. OF CALIF., SANTA CRUZ

UNIV. OF MONTANA

UNIV. OF OREGON

UNIV. OF SOUTHERN CALIFORNIA

UNIV. OF UTAH

UNIV. OF WASHINGTON

WASHINGTON STATE UNIVERSITY

These supporting institutions contribute to the cost of publication of this Journal, but they are not owners or publishers and have no responsibility for its contents or policies.

See inside back cover or www.pjmath.org for submission instructions.

The subscription price for 2011 is US \$420/year for the electronic version, and \$485/year for print and electronic.

Subscriptions, requests for back issues from the last three years and changes of subscribers address should be sent to Pacific Journal of Mathematics, P.O. Box 4163, Berkeley, CA 94704-0163, U.S.A. Prior back issues are obtainable from Periodicals Service Company, 11 Main Street, Germantown, NY 12526-5635. The Pacific Journal of Mathematics is indexed by Mathematical Reviews, Zentralblatt MATH, PASCAL CNRS Index, Referativnyi Zhurnal, Current Mathematical Publications and the Science Citation Index.

The Pacific Journal of Mathematics (ISSN 0030-8730) at the University of California, c/o Department of Mathematics, 969 Evans Hall, Berkeley, CA 94720-3840, is published monthly except July and August. Periodical rate postage paid at Berkeley, CA 94704, and additional mailing offices. POSTMASTER: send address changes to Pacific Journal of Mathematics, P.O. Box 4163, Berkeley, CA 94704-0163.

PJM peer review and production are managed by EditFLOW ${ }^{\mathrm{TM}}$ from Mathematical Sciences Publishers.

PUBLISHED BY PACIFIC JOURNAL OF MATHEMATICS

at the University of California, Berkeley 94720-3840

A NON-PROFIT CORPORATION

Typeset in IATEX

Copyright $(2011$ by Pacific Journal of Mathematics 


\title{
PACIFIC JOURNAL OF MATHEMATICS
}

\author{
Volume $253 \quad$ No. $1 \quad$ September 2011
}

Singularities of the projective dual variety

ROLAND ABUAF

Eigenvalue estimates for hypersurfaces in $\mathbb{H}^{m} \times \mathbb{R}$ and applications

Pierre BÉRARD, Philippe CASTILlon and Marcos CaVAlCante

Conformal Invariants associated to a measure: Conformally covariant operators

Sun-Yung A. Chang, Matthew J. Gursky and Paul Yang

Compact symmetric spaces, triangular factorization, and Cayley coordinates

DEREK HABERMAS

Automorphisms of the three-torus preserving a genus-three Heegaard splitting

JESSE JOHNSON

The rationality problem for purely monomial group actions

HiDETAKA KiTAYAMA

On a Neumann problem with $p$-Laplacian and noncoercive resonant nonlinearity

Salvatore A. Marano and Nikolaos S. Papageorgiou

Minimal ramification in nilpotent extensions

NADYA MARKIN and STEPHEN V. ULLOM

Regularity of weakly harmonic maps from a Finsler surface into an $n$-sphere

XIAOHUAN MO and LIANG ZHAO

On the sum of powered distances to certain sets of points on the circle

NikOlai NikOlOV and RAFAEL RAFAilov

Formal geometric quantization II

PAUL-ÉMILE PARADAN

Embedded constant-curvature curves on convex surfaces

HAROLD ROSENBERG and MATTHIAS SCHNEIDER

A topological construction for all two-row Springer varieties 